\title{
Das Existo und die Natur des Geistes
}

Zwei Fragen sind es, die den Überlegungsgang der Zweiten Meditation bestimmen. Existiert etwas, an dem zu zweifeln nicht die geringste Gelegenheit besteht? Diese erste Frage findet ihre bejahende Antwort in der Einsicht des Denkers, daß er selbst so etwas ist. Seine zweite Frage ist dann, wer oder was er selbst ist.

Die Ziele, die Descartes in seinem Überlegungsgang verfolgt, sind weit vielfältiger, als diese beiden Leitfragen allein es vermuten lassen. Mit dem Beweis seiner eigenen Existenz gewinnt der Denker ein Paradigma dessen, was es heißt, einen im höchsten Maße klaren und deutlichen Gedanken zu haben; dies ist ein entscheidender Schritt in Richtung auf die später formulierte und begründete Wahrheitsregel. Zudem werden Prämissen des Beweises für die reale Verschiedenheit der körperlichen und der geistigen Substanz bereitgestellt, der erst in der Sechsten Meditation geführt wird. Weitere Ziele, die Descartes verfolgt, haben es damit zu tun, die traditionelle Schulmetaphysik mit der gebotenen Vorsicht auch im Hinblick auf das Wesen der Selbstkenntnis des Intellekts methodisch und inhaltlich als unterlegen erkennbar werden zu lassen ${ }^{1}$, den Intellekt als das eigentliche menschliche Erkenntnisorgan und andere Vermögen als epistemisch minderwertig zu erweisen, den Leser durch ein Beispiel auf seine Konzeption der körperlichen Substanz einzustimmen, ${ }^{2}$ und einiges andere mehr. - Im folgenden werde ich mich auf Descartes' Auseinander- 
setzung mit den beiden Leitfragen konzentrieren und Nebenthemen wie die gerade genannten weitestgehend beiseite lassen.

\section{Die Gewißheit meiner Existenz}

Die Art und der Umfang des Großen Zweifels, in dem der Denker sich zu Beginn der Zweiten Meditation befindet, ist durch die TäuschergottProbe bestimmt: Als gewiß soll nur das gelten, das nicht einmal unter der Annahme in Zweifel gezogen werden kann, daß es einen höchst mächtigen Täuscher gibt, der den Denker in allem täuscht, in dem ihm dies möglich ist (AT VII 27). „Es ist gewiß, daß $p$ “ heißt für den Denker: „Selbst unter der Annahme der Existenz eines solchen Täuschergottes kann ich nicht in Zweifel ziehen, daß $p$ ". Was ist ihm in diesem Sinne gewiß? Nur etwas, in dessen Negation ihm eine offenkundige Widersinnigkeit [repugnantiam ... manifestam, AT VII 36] erkennbar ist. Alles andere hingegen soll ihm zunächst einmal als falsch gelten.

Zur Illustration des Umfangs seines Großen Zweifels nennt der Denker folgende Beispiele dafür, was ihm nun alles als falsch gilt: daß auf sein Gedächtnis Verlaß ist; daß er Sinne hat; und daß es Körper, den Himmel, die Erde oder Geister [mentes] gibt (AT VII 24 f.). Daß er an dieser Stelle die Existenz von Geistern in Abrede stellt, ist kein Lapsus, sondern hat eine Pointe, die kurz darauf klar wird (AT VH 27): Ein präziser Sinn des Wortes "Geist" ist ihm an diesem Punkt noch unbekannt. Er wird erst dann verstehen, was "Geist" eigentlich beinhaltet, wenn er eingesehen haben wird, daß er selbst ein denkendes Ding ist, und begriffen haben wird, worin die unüberbietbare Gewißheit dieser Einsicht ihren Grund hat.

Nun fragt sich der Denker, wie es um seine eigene Existenz steht: Kann er sie in Zweifel ziehen? Hat er sie womöglich bereits dadurch in Zweifel gezogen, daß er daran zweifelt, daß er Sinne und einen Körper hat? Mit diesen Fragen ist der entscheidende Teil des Überlegungsgangs erreicht, in dem der Denker seine erste Gewißheit erreicht: daß er existiert. Die anschließende Überlegung wird von Descartes als eine Abfolge von versuchsweisen Annäherungen an diese Einsicht entwickelt.

\section{Erster Überlegungsschritt:}

Wenn ich mich dazu gebracht habe anzunehmen, daß nichts in der Welt existiert, dann habe ich gewiß existiert.

Dies ist eine bedingte Gewißheit; ihre Schwäche hängt damit zusammen, daß sie die Vergangenheit betrifft. Um ihre Bedingung als erfüllt anzuneh- 
men, müßte der Denker sich auf sein Gedächtnis verlassen, was aber, wie gerade gesehen, in seinem Großen Zweifel nicht zulässig ist.

\section{Zweiter Überlegungsschritt:}

Wenn mich jetzt ein Täuschergott täuscht, dann existiere ich ohne Zweifel.

Auch dies ist eine bedingte Gewißheit; jedoch ist ihre Bedingung bemerkenswerterweise gerade unter Voraussetzung derjenigen hypothetischen Unterstellung erfüllt, die den Großen Zweifel ausmacht. Dennoch, auch hierin findet der Denker noch nicht das, was er sucht. Denn die Hypothese, daß es einen Täuschergott gibt, kann ihrerseits selbst in Zweifel gezogen werden. Sie dient dem Denker als Probierstein für Gewißheit, nicht als eine Prämisse, die ihren Folgerungen Gewißheit verleihen könnte. Interessanterweise stellt der Denker nun nicht die weitere Überlegung an: „Erst recht gilt auch: Wenn mich jetzt kein Täuschergott täuscht, dann existiere ich ohne Zweifel“, woraus er mit dem zweiten Schritt zusammen die Gewißheit seiner Existenz erlangen könnte. Stattdessen:

Dritter Überlegungsschritt:

Auch wenn mich stets ein Täuschergott täuscht, läßt sich folgendes nicht in Zweifel ziehen: Wann immer ich denke, daß ich existiere, existiere ich.

Im Lichte der Cartesischen Konzeption von Gewißheit heißt dies:

Es ist gewiß: Ich existiere, wann immer ich denke, daß ich existiere. Und daraus folgt:

Wann immer gewiß ist, daß ich denke, daß ich existiere, ist gewiß, daß ich existiere.

Descartes läßt seinen Denker das Ergebnis dieser Überlegungen so zusammenfassen: „Nachdem alles übergenug durchdacht ist, ist schließlich festzuhalten, daß dieser Satz Ich bin, ich existiere, sooft ich ihn ausspreche oder im Geist erfasse, erwiesenermaßen ${ }^{3}$ [necessario] wahr ist" (AT VII 25).

Damit ist für Descartes in den Meditationen der Beweis vollendet; vom nächsten Satz an spricht der Denker so, als sei seine Existenz erwiesen. Die Erkenntnis, daß er existiert, nennt er im selben Satz die gewisseste und evidenteste von allen.

3 Mit dieser Übersetzung von „necessario“ folge ich dem Hinweis von Rainer Specht (1996, 9), daß dieser Terminus gelesen werden darf als: durch Beweis gesichert. 


\section{Cogito, ergo sum und Ego sum, ego existo}

Was sich in der geschilderten Argumentation nicht findet, ist das berühmte „Ich denke, also bin ich“, das Descartes an einigen anderen Stellen seines Werks, vor und nach Abfassung der Meditationen, formuliert. Im Discours von 1637 bezeichnet Descartes (AT VI 33) „cette vérité: je pense, donc je suis“ als das erste Prinzip der Philosophie, die er gesucht habe; in den Principia von 1644 heißt es (AT VIII-1 7): „haec cognitio, ego cogito, ergo sum“ sei die erste und gewisseste aller Erkenntnisse, die jedem begegne, der der Ordnung gemäß philosophiere. Selbst im Zusammenhang der Meditationen, nämlich in den Erwiderungen auf Einwände gegen die Zweite Meditation, verwendet er die Wendung ,ego cogito, ergo sum, sive existo“ (AT VII 140). - Dies mag man als Hinweise darauf werten, daß er anscheinend keinen bedeutsamen Unterschied zwischen „Ich existiere“ und „Ich denke, also existiere ich“ macht. Dennoch, der Wortlaut der Zweiten Meditation ist eindeutig; das Ergebnis der dort entwickelten Argumentation ist „Ich existiere“. Dies ist, in den Meditationen zumindest, die erste und gewisseste Erkenntnis des Denkers.

\section{Schluß oder Intuition?}

Doch welches ist eigentlich die logische Struktur der Argumentation, mit der der Denker zu dieser Konklusion gelangt? Ja, es ist zu fragen: Ist es überhaupt eine Konklusion? Erreicht er die Einsicht, daß er existiert, überhaupt mittels eines Schlusses? Oder erreicht er sie vielmehr mittels einer selbstevidenten Intuition, die sich schließlich in seinem Denken einstellt, nachdem er mehrere vorbereitende Überlegungen zum Zusammenhang von Denken und Existenz angestellt hat - Überlegungen, die ihm zu dieser intuitiven Einsicht zwar den Weg bahnen, aber eben nicht in der Weise, daß er sie aus ihnen schlußfolgert?

Wir finden bei Descartes Hinweise, die sich zugunsten beider Deutungen geltend machen lassen. Für die Schlußfolgerungsdeutung sprechen viele Stellen, an denen er in diesem Zusammenhang mit Selbstverständlichkeit inferentielle Terminologie verwendet. So heißt es in der Vierten Meditation, er habe (in der Zweiten Meditation) untersucht, ob überhaupt irgend etwas in der Welt existiert, und erkannt, daß gerade daraus, daß er dies untersuche, evidentermaßen folge [evidenter sequi], daß er existiert (AT VII 58). In den Fünften Erwiderungen sagt er, er könne aus der Tatsache, daß er denkt, er gehe spazieren, bestens schlußfolgern [optime inferre], daß es einen Geist gibt, der diesen Gedanken hat (AT VII 352). 
Anders klingt, was Descartes in den Zweiten Erwiderungen darlegt: „Wenn wir bemerken, daß wir denkende Dinge sind, so ist das eine erste Erkenntnis, die nicht aus irgendeinem Syllogismus geschlußfolgert wird; und auch wenn jemand sagt Ich denke, also bin oder existiere ich, so deduziert er seine Existenz nicht vermittels eines Syllogismus aus dem Denken, sondern erkennt sie gleichsam wie eine durch sich selbst gewußte Sache vermittels einer einfachen Intuition des Geistes" (AT VII 140). Descartes führt dafür folgendes Argument an: Handelte es sich um einen Syllogismus, so müßte der betreffende Denker vorgängiges Wissen über die zusätzliche allgemeine Prämisse haben, daß alles, was denkt, existiert; dieses Wissen gewinne er aber erst dadurch, daß er an sich selbst erlebt, daß es nicht sein kann, daß er denkt, ohne zu existieren. - Diese Stelle spricht zwar deutlich für die Intuitionsdeutung, was jedoch den etwaigen inferentiellen Charakter des Ich denke, also bin ich angeht, so ist zu beachten, daß Descartes hier nur bestreitet, daß es ein Syllogismus ist. Er schließt damit nicht aus, daß es sich um eine Schlußfolgerung anderer Art handelt - um eine, die kein Syllogismus ist. Die Hoffnung, die Intuitions- und die Schlußfolgerungsdeutung miteinander zu vereinbaren, ist dadurch nicht zerstört. Der Schluß auf die eigene Existenz müßte allerdings ein nicht-syllogistischer sein.

Ein weiterer Beleg für die Intuitionsdeutung: An den Marquis von Newcastle schreibt Descartes im April 1648, das Wissen um die Wahrheit der Proposition Ich denke, also bin ich sei nicht das Werk vernünftigen Schließens [raisonnement], sondern ein intuitives Wissen (AT V 138). Bemerkenswert ist, daß hier anscheinend jede Art des Vernunftschließens, nicht nur die syllogistische, in Abrede gestellt wird. Spricht dies nicht endgültig gegen die Vereinbarkeit von Schluffolgerungs- und Intuitionsdeutung? Nein, denn das, was Descartes in diesem Brief als intuitiv gewußt bezeichnet, ist ja selbst gerade eine Proposition, in der es um eine Schlußfolgerung geht.

Eine Herausforderung an jede Auslegung des Cartesischen Beweises eingangs der Zweiten Meditation ist es demnach, erklärlich zu machen, wie Descartes die Gewißheit seiner Existenz sowohl als auf einer einfachen Intuition beruhend betrachten kann, als auch als etwas, das er aus seinem Denken erschließt. Der Kürze halber werde ich im folgenden den Inhalt dessen, was der Denker denkt, wenn er justament unter den besonderen epistemischen Gegebenheiten des Großen Zweifels denkt, daß er existiert,

4 Der Hinweis auf diese spezielle Situation ist keine überflüssige Akribie, sondern soll darauf aufmerksam machen, daß der Denker in anderen epistemischen Gegebenheiten, in denen er es sich nicht versagt, auf Wissen über sich selbst zurückzugreifen, mit demselben Satz, „Ich bin, ich existiere", einen anderen Gedanken verbinden mag. 
als Existo bezeichnen (und den entsprechenden Gedanken als seinen ExistoGedanken).

Ein vielbeachteter Vorschlag zu einer Analyse der Argumentationsstruktur, in dem die Schlußfolgerungs- und Intuitionsdeutung kombiniert werden, stammt von Peter Markie (1992). ${ }^{5}$ Er vertritt die Auffassung, daß Descartes in den Regulae zwei Arten von intuitivem Wissen zulasse. Intuitives Wissen im engeren Sinn haben wir, wenn eine Proposition „durch den reinen und aufmerksamen Geist so leicht und deutlich erfaßt wird, daß keinerlei Zweifel an dem, was wir verstehen, übrig ist" (AT X 368); solche Propositionen sind erste Prinzipien. Als intuitiv gewußt im weiteren Sinn bezeichnet Markie (nicht Descartes) die Wahrheit solcher Propositionen, „die aus ersten Prinzipien unmittelbar gefolgert werden“ (AT X 370). Propositionen, zu denen man von ersten Wahrheiten nur durch mehrere Folgerungsschritte gelangt, werden durch Deduktion gewußt. Markies Vorschlag zur Vereinbarkeit der beiden Deutungen besagt nun folgendes: Descartes könne im Hinblick auf das Existo sowohl davon sprechen, es werde gefolgert, als auch, es werde intuitiv gewußt, denn es sei ja ein intuitives Wissen im weiteren Sinn, da es unmittelbar aus einer Prämisse, Ich denke, gefolgert werde, die selbst wiederum nicht durch Folgerung, sondern durch Intuition im engeren Sinn als wahr eingesehen werde.

Markies Vorschlag hat einige Schwächen. Die Proposition Ich denke müßte dann das erste Prinzip sein, aus dem der Denker seine Existenz folgert; das paßt aber, wie gesehen, nicht zum Text der Zweiten Meditation. Zweitens nennt Descartes die Intuition, der der Denker sein Wissen um die eigene Existenz verdankt, eine einfache Intuition. Doch Intuitionen in Markies weiterem Sinn sind offenkundig komplex; sie bestehen aus zwei Komponenten: einer intuitiven Erkenntnis im engeren Sinn und einer Folgerung aus ihr. Drittens wird durch Markies Vorschlag nicht erklärlich, weshalb Descartes ausdrücklich den Existo-Gedanken als denjenigen nennt, durch den der Denker sich seiner eigenen Existenz gewiß wird. ${ }^{6}$ - Es erscheint mithin lohnend, nach einer anderen Auslegung zu suchen.

$5 \mathrm{Zu}$ einem anderen Vorschlag dieser Art vgl. S. Gaukroger $(1995,341)$, der an dieser Stelle die Auffassung vertritt, für Descartes sei jede Intuition ein Schluß. Plausibler ist hingegen, was er im selben Buch an früherer Stelle (117 f.) ausführt: daß für Descartes ein Schluß unter besonderen Bedingungen auch eine Intuition sein kann.

6 Zudem setzt Markie voraus, der Denker erkenne durch Intuition, daß er denkt. Auch dies erscheint, als Descartes-Deutung, unhaltbar. Vom eigenen Denken hat der Denker keine Erkenntnis (geschweige denn vermittels Intuition), sondern schlicht Bewußtsein. Siehe dazu Kemmerling (1996, Kap. 5). 


\section{Das Existo-Argument}

In der philosophischen Diskussion ist es üblich, Descartes' Versuch, die eigene Existenz als gewiß zu erweisen, als „das Cogito-Argument“ zu bezeichnen. Diese Charakterisierung suggeriert, es finde sich in Descartes' Werk immer ein und dieselbe Überlegung, die zur Gewißheit über die eigene Existenz führt. Doch Descartes' Schriften enthalten Hinweise auf unterschiedliche Varianten, deren argumentative Äquivalenz keineswegs offenkundig ist; Beispiele sind: „Ich untersuche, ob überhaupt irgendetwas in der Welt existiert, also bin ich“ (AT VII 58); „Ich glaube, daß ich spazierengehe, also gibt es einen Geist, der das glaubt“" (AT VII 352); „Eine Sache, die denkt, kann nicht nicht existieren“ (AT VII 473); „Es ist nicht möglich zu zweifeln, daß zumindest eine zweifelnde oder denkende Substanz existiert“ (AT VII 537); „Es ist widersinnig zu glauben, daß das, was denkt, zur selben Zeit, zu der es denkt, nicht existiert“ (AT VIII-1 7); „Es ist nicht möglich, daß das, was denkt, nicht existiert“ (AT VIII-1 8); und schließlich: „Du kannst nicht bezweifeln, daß du, der du zweifelst, existierst" (AT X 515).

Die Rede von dem Cartesischen Cogito-Argument ist aber - jedenfalls im Hinblick auf den Überlegungsgang eingangs der Zweiten Meditation auch noch in einer weiteren Hinsicht irreführend: insofern nämlich, als sie nahelegt, in dem Satz, der den gewißheitsstiftenden Gedanken ausdrückt, müsse das eigene Denken explizit thematisiert werden.

Doch dies ist anscheinend nicht der Fall. Eine besondere Raffinesse des Beweises, der eingangs der Zweiten Meditation geführt wird, liegt gerade darin, daß der Gedanke, durch den der Denker sich seiner eigenen Existenz gewiß wird, schlicht der von „Ich existiere" ausgedrückte selbst ist. Im dritten Schritt wurde dem Denker klar, daß das Denken des Existo-Gedankens allein schon ausreicht, um sich seiner Existenz gewiß zu sein. Genau diese Besonderheit des Existo-Gedankens läßt Descartes seinen Denker als das Ergebnis seiner Überlegung festhalten. Nennen wir das Argument, mit dem der Denker in der Zweiten Meditation zur Gewißheit seiner eigenen Existenz gelangt, also passenderweise: das Existo-Argument.

Betrachten wir noch einmal das Ergebnis des dritten vorbereitenden Überlegungsschritts:

Wann immer gewi $\beta$ ist, daß ich denke, daß ich existiere, ist gewiß, daß ich existiere.

Die Hervorhebung soll verdeutlichen, warum es so scheinen mag, als reiche es zur Erlangung der Gewißheit über die eigene Existenz mit Hilfe 
des Existo-Arguments nicht aus, daß der Denker den Existo-Gedanken hat; als müsse es ihm darüber hinaus auch gewiß sein, daß er diesen Gedanken hat. Wie könnte ihm dies gewiß sein? Als Antwort könnte vorgeschlagen werden: Nun, durch das Denken dieses Gedankens selbst. Zu ihrer exegetischen Stützung könnte ins Feld geführt werden, daß Descartes (z. B. in AT VII 246) eine generelle Transparenzthese vertritt, der zufolge wir uns jedes geistigen Akts, der in uns statthat, und mithin auch jedes Gedankens, den wir haben, bewußt sind (und wer sich eines Gedankens bewußt ist, verfügt ipso facto über Gewißheit, daß er ihn hat). - Doch selbst wenn diese vielfach bestrittene Transparenzthese wahr wäre, dürfte der Denker sie an diesem Punkt seines Überlegungsgangs als wahr voraussetzen? Dies dürfte er, im Lichte seiner eigenen Festsetzung dazu, was er als wahr gelten läßt, nur dann, wenn selbst ein Täuschergott nicht bewirken könnte, daß der Denker Gedanken hat, die ihm nicht bewußt sind. Aber es liegt keine offenkundige Widersinnigkeit darin, daß ein Täuschergott dies vermag. Und so ist häufig gegen Descartes eingewandt worden, im Schluß des Denkers auf seine Existenz klaffe eine Begründungslücke: Es fehle ein Argument für die Unbezweifelbarkeit seines momentanen Denkens (vgl. z. B. Frankfurt 1970, 110 f. und Wilson 1978, 58 ff.).

$\mathrm{Da} 3$ solch ein Argument in der Zweiten Meditation jedoch nicht fehlt, läßt sich einsehen, wenn beachtet wird, wonach der Denker sucht: nach einem Gedanken nämlich, der eine Existenz-Proposition zum Inhalt hat, die ihm unbezweifelbar ist. Das Kriterium für Unbezweifelbarkeit ist, daß dem Denker die manifeste Widersinnigkeit der Negation des Gedankens erkennbar ist. Diesem Kriterium genügt der Existo-Gedanke, und zwar ohne jede zusätzliche Prämisse - auch ohne die Prämisse, daß dem Denker jeder seiner Gedanken bewußt ist. Es reicht, daß ihm, wenn er den ExistoGedanken hat, ebenfalls die manifeste Widersinnigkeit der Annahme erkennbar ist, er habe diesen Gedanken nicht. Und dazu benötigt er keinen zusätzlichen Gedanken, geschweige denn eine allgemeine Prämisse; es reicht, den Existo-Gedanken selbst zu denken.?

Auch ist es nicht nötig, daß der Denker schon genau versteht, welchen spezifischen Sinn (d. h. welchen exakten kognitiven Gehalt) er mit „ich“ verbinden muß; es reicht, daß er mit dem Wort beim Denken des ExistoGedankens irgendeinen passenden Sinn verbindet, der seiner Bedeutung (d. h. seinem semantischen Gehalt) entspricht und den besonderen Gege-

7 Anders gesagt: Im Denken des Gedankens selbst hat der Denker simples (wenn auch nicht reflektierendes) Bewußtsein dessen, daß er diesen Gedanken denkt. Daß dies jedenfalls Descartes' Auffassung ist, habe ich in Kemmerling (1996, 217-232) näher ausgeführt. 
benheiten des Großen Zweifels angemessen ist. ${ }^{8}$ Indem Descartes den Denker sein Ergebnis metasprachlich (als eines über die Wahrheit des Satzes „Ich existiere") formulieren läßt, läßt er offen, welchen spezifischen Sinn dieser beim Denken des Inhalts dieses Satzes mit dem Wort „ich“verbindet. Das Ergebnis gilt für jeden beliebigen zulässigen Sinn dieses Wortes - d. h. für jeden kognitiven Gehalt, der im Einklang steht mit der Bedeutung dieses Wortes. Vorausgesetzt wird dabei allerdings, daß dem Denker trotz seines Großen Zweifels ein zulässiger Sinn von „ich“ verfügbar ist; und wir werden uns als nächstes der Frage zuwenden, welcher Sinn das ist.

Descartes beeilt sich, gleich im Anschluß an den Existenz-Beweis deutlich zu machen, daß der Denker nun zwar weiß, daß er existiert, aber immer noch nicht, wer oder was er ist. Im Gegenteil, erst der Umstand, daß er auf eine sehr bestimmte Weise Gewißheit seiner Existenz erlangt hat, ermöglicht es dem Denker, auch zu einem präzisen und für seine metaphysischen Zwecke hilfreichen Sinn des Wortes „ich“ zu gelangen. In der Zweiten Meditation findet, wie wir sehen werden, also auch hinsichtlich des kognitiven Gehalts von „ich“ ein Erkenntnisfortschritt in drei Stufen statt. Eingangs verbindet der Denker mit dem Wort, „ich“ einen unreflektierten Sinn, der unbestimmt bleibt. Im Beweis selbst nimmt dieses Wort einen ausnehmend speziellen und instabilen Sinn an, der mit Raffinesse auf die besonderen epistemischen Zwecke zugeschnitten ist. (Die Überwindung dieses einmaligen Zweifels erfordert, wie wir sehen werden, einen sozusagen einmaligen Sinn des Wortes „ich“.) Und dank diesem Beweis gelangt der Denker schließlich zu einem präzisen und stabilen Sinn, den er im weiteren Verlauf der Meditationen mit dem Wort „ich“ verbinden kann. ${ }^{9}$

Als was nun kann der Denker sich selbst begreifen, wenn er sich in der Extremsituation des Großen Zweifels befindet? Nicht als einen Körper oder als etwas, das einen Körper hat; nicht als etwas, das durch Vergangenes bestimmt ist. All dies ist, wie wir gesehen haben, dem Großen Zweifel ausgesetzt. Der Denker ist sich selbst, in dem Moment, in dem er seine eigene Existenz als unbezweifelbar einsieht, ausschließlich als einer gegeben, der denkt - und zwar just den Gedanken denkt, den er in diesem gegenwärtigen Moment denkt. Wessen er sich beim Denken des ExistoGedankens gewiß ist, läßt sich entsprechend so wiedergeben:

8 Zur Unterscheidung zwischen dem kognitiven Gehalt (Sinn) und dem semantischen Gehalt (Bedeutung) von ,ich“ siehe Kemmerling (1996, 108-117).

9 In den Meditationen geht es Descartes eben auch darum, dem Leser an Beispielen vorzuführen, wie Begriffsbildung in der Philosophie vor sich gehen soll. Vgl. dazu auch die Beiträge von Hüttemann (Kapitel 9) und Schütt (Kapitel 8) in diesem Band. 


\section{(Existo) Der Denker dieses Gedankens existiert,}

wobei der hervorgehobene Ausdruck auf das jeweilige Gedankenvorkommnis von (Existo) selbst verweist. ${ }^{10}$ Dieser Gedanke ist in folgendem Sinne wahrheitsautonom: Der bloße Umstand, daß er gedacht wird, reicht dafür aus, daß er wahr ist. (Anders gesagt: in jeder Welt, in der er gedacht wird, ist er wahr - gleichgültig, was außerdem in dieser Welt der Fall ist oder nicht der Fall ist. Müßte eine Welt, in der dieser Gedanke wahr ist, denn noch etwas enthalten außer dem singulären Ereignis des Gedachtwerdens dieses einen Gedankens? - Dies scheint mir Descartes' faszinierende Ausgangsidee zu sein.) Ein wahrheitsautonomer Gedanke ist in dem von Descartes bemühten Sinn gewißheitsstiftend: Seine Negation ist jedem, der ihn denkt (wenn ihn denn jemand denkt), als eine offenkundige Widersinnigkeit erkennbar.

Ist das selbstbezügliche (Existo) ein brauchbarer Kandidat zur Erläuterung des Existo-Gedankens? Nun, es paßt trefflich zur Intuitionsdeutung: Wird dieser Gedanke, entsprechend der oben genannten Definition von „intuitio“ in den Regulae, von einem reinen und aufmerksamen Geist erfaßt, dann bleibt kein Zweifel an der Wahrheit dessen zurück, was da erfaßt wird. Eine Schwäche dieser Interpretation ist allerdings, daß dieser Gedanke nichts Inferentielles an sich hat; (Existo) paßt demnach nicht zur Schlußfolgerungsdeutung. Doch es gibt einen dem (Existo) inhaltlich eng verwandten Gedanken, durch den sich auch dieses interpretative Desiderat erfüllen läßt:

$\left(\right.$ Existo $\left.^{+}\right)$Der Denker dieses Gedankens [hat diesen Gedanken und folglich gilt: er] existiert.

Die eckigen Klammern machen kenntlich, worin dieser Gedanke eine Anreicherung des (Existo) ist: Hierin steckt das „,cogito, ergo“. Wird (Existo ${ }^{+}$ gedacht, so wird der Inhalt von „Ich denke, also ..." mitgedacht. Welchen Gedanken hätte der Denker, wenn er an dieser Stelle nichts anderes dächte als: daß er denkt? Bei der Antwort auf diese Frage ist zu beachten, daß „Ich denke" keinen vollständigen Gedanken ausdrückt; bestenfalls ist es eine elliptische Charakterisierung eines Gedankens, aus der selbst nicht hervorgeht, welcher Gedanke es ist. Allein deshalb schon kann „Ich denke“ nicht die Prämisse eines Schlusses sein - genauso wenig, wie etwa „Ich sorge“ oder „Ich löse“. Im Lichte von $\left(E_{\text {Existo }}^{+}\right)$ist es naheliegend, den durch „Ich denke“ nur unvollständig charakterisierten Gedanken so zu spezifizieren:

10 Eine peniblere Version von (Existo) lautet also: „Wer oder was auch immer dieses Gedankenvorkommnis hat, existiert." - Zu einer ausführlicheren Begründung dieser Deutung vgl. Kemmerling (1996, 93-121). 
(Cogito) Der Denker dieses Gedankens hat diesen Gedanken.

Folgt (Existo) aus (Cogito)? Nein, denn (Cogito) kann wahr sein, wenn (Existo) nicht wahr ist. Beide Gedanken sind zwar unweigerlich wahr, wenn sie gedacht werden; aber jeder von ihnen ist (der Selbstbezüglichkeit wegen) eben auch nur dann wahr, wenn $e r$ gedacht wird. Und daraus, daß der erste gedacht wird, folgt nicht, daß der zweite gedacht wird, und erst recht nicht, daß beide vom selben Denker gedacht werden. - Ein Folgerungszusammenhang kann nur dadurch gestiftet werden, daß beide Gedanken gleichsam zu einem verschmolzen werden. ${ }^{11}$ Das Ergebnis dieser ,Verschmelzung', samt Einbeziehung der Folgerungsbeziehung, ist $\left(\right.$ Existo $\left.^{+}\right)$, ein Gedanke, der somit auch als Cogito, ergo sum bezeichnet zu werden verdient. Zu beachten ist dabei jedoch, daß auch $\left(E_{\left.x i s t 0^{+}\right)}\right.$nicht aus (Cogito) folgt; kein Schluß im üblichen Sinn führt von einem dieser drei Gedanken zu einem der beiden anderen. Kurz, wer in der Zweiten Meditation nach einer gültigen Variante des Cogito-Arguments sucht, findet sie eher in (Existo $\left.{ }^{+}\right)$selbst als in dem Übergang von (Cogito) zu (Existo) oder zu (Existo ${ }^{+}$.

$\left(E\right.$ sisto $\left.^{+}\right)$ist wahrheitsautonom und mithin durch einen Akt der Intuition als wahr zu erkennen; zudem paßt dieser Gedanke sehr gut zur Schlußfolgerungsdeutung. Im Hinblick auf diesen Gedanken ist mithin das exegetische Desiderat der Vereinbarkeit beider Deutungen erfüllt. Da (Existo $\left.{ }^{+}\right)$ mit einigem Recht auch als Cogito, ergo sum bezeichnet werden darf, liefert es außerdem zugleich eine Erklärung dafür, weshalb Descartes bei der Frage, was die erste Gewißheit seines Denkers sei, gelegentlich keinen Unterschied zwischen „Ich existiere“ und „Ich denke, also bin ich" macht.

Unsere Betrachtung legt mithin folgendes nahe. Während Descartes im Discours und an anderen Stellen die Unbezweifelbarkeit von (Existo ${ }^{+}$), alias Cogito, ergo sum, hervorhebt, läßt er seinen Denker in dem Überlegungsgang eingangs der Zweiten Meditation nach drei vorbereitenden Schritten zu der Einsicht gelangen, daß der einfachere Gedanke (Existo) ausreicht, um sich der eigenen momentanen Existenz zu vergewissern. Dies geschieht in einem Akt der Intuition. - Natürlich hielt Descartes, wie aus vielen Stellen hervorgeht, auch den Schluß

Ich denke.

Ich existiere.

11 Aus seinen Ausführungen zur siebten Regel geht hervor, daß Descartes auch komplexen Gedanken, deren Inhalt einen Folgerungsschritt umfaßt, den Rang einer Intuition zubilligt: „das Ganze scheint mir gleichzeitig durch Intuition erkannt zu werden“ (AT X 388). 
für gültig und für einen zwingenden Beweis. Aber solch ein regulärer Schluß vom Denken auf das eigene Existieren, mit separater Prämisse und Konklusion, ist dem Denker an dieser Stelle der Zweiten Meditation durch seine besondere epistemische Situation verwehrt, in der er sich derartig starke Erkenntnisansprüche auferlegt, daß er sich selbst nur als der Denker des jeweiligen Gedankens gegeben ist, den er gerade hat.

Im Lichte dieser Rekonstruktion ist das Cartesische Argument, das eingangs der Zweiten Meditation zu finden ist, scheinbar nicht dem berühmten Einwand ausgesetzt, den u. a. Lichtenberg, Nietzsche und Russell vorgebracht haben. ${ }^{12}$ "Cogito" sei schon zu viel, sobald man es durch „Ich denke“ übersetze, konstatiert Lichtenberg und empfiehlt: „Es denkt“ sollte man sagen, so wie man sagt: „Es blitzt“ (Lichtenberg 1969, 412). Nietzsche hält selbst ein „Es denkt“ und schließlich sogar das passivische „cogitatur“ für epistemisch zu voraussetzungsreich (Nietzsche 1968, Bd. VI-2, 24 ff. und 1970 Bd. VII-3, 371 ff.). Und Russell bemerkt, „Ich denke“ sei Descartes' ultimative Prämisse, aber das Wort „ich“ darin sei illegitim; er hätte die Prämisse so formulieren sollen: „Es gibt Gedanken“ (Russell 1945, 567). - Zwar setzt dieser Einwand beim Cogito-Gedanken an und betrifft damit, in der vorgebrachten Form, nicht das Existo-Argument. Dennoch, der springende Punkt dieses Einwands trifft auch das (Existo): Descartes setzt darin jedenfalls eines unbegründet voraus: daß es zu jedem Gedankenvorkommnis etwas gibt, an oder in dem es vorkommt.

\section{Sum res cogitans}

Der Denker glaubt nun zwar zu wissen, daß er ist; aber noch nicht, wer oder was er ist. Indem er an sich ausschließlich als einen Denkenden dachte, vermochte er es (jedenfalls zu seiner eigenen philosophischen Zufriedenheit - wenn auch nicht zu der von Lichtenberg \&Co), seine Existenz als gewiß einzusehen. Daraus versucht er nun, eine weitere Gewißheit zu gewinnen: daß er etwas, eine Sache (philosophisch gesprochen: eine Substanz), ist, die Gedanken hat.

$\mathrm{Da}$ er, dessen Existenz nun erwiesen ist, jedenfalls eine Substanz ist, ist dem Denker offenbar keinerlei eigener Begründung bedürftig. „Aber das heißt, unsern Glauben an den Substanzbegriff schon als, wahr a priori' anzusetzen“, moniert Nietzsche (1970, Bd. 2, 215), wiederum völlig zurecht.

12 Neuerdings hat F. Dretske (2003) diesen Einwand verschärft: Man könne zwar wissen, was man denkt, aber nicht, daß man es denkt. 
Daß dasjenige, das Gedanken hat, eine Substanz ist, ist eine weitere unbegründete Voraussetzung, die Descartes in seinem Überlegungsgang macht. Häufig wird diese Voraussetzung als eine des vorausgegangen Beweises der eigenen Existenz betrachtet, so z. B. bei Kenny $(1968,60)$ und Dicker (1993, 53 ff.). Mit Hinblick auf die Zweite Meditation ist dies jedoch, wie wir gesehen haben, nicht haltbar. Das Existo-Argument selbst ist auf diese Voraussetzung nicht angewiesen. Dennoch, unmittelbar anschließend wird sie gemacht. An einer späteren Stelle, an der Descartes auf sie zu sprechen kommt, begründet er sie nicht, sondern verstärkt sie nur: Es sei gewiß, bemerkt er in den Dritten Erwiderungen, daß es einen Gedanken nicht ohne denkende Substanz geben könne; wie es ja überhaupt keinen Akt und kein Akzidens geben könne: ohne eine Substanz, in der sie sind (AT VII 175 f.). $\mathrm{Ob}$ und in welchem Maße diese Voraussetzung philosophisch verfänglich ist, hängt natürlich davon $\mathrm{ab}$, was für eine Substanz-Konzeption dahintersteckt. ${ }^{13}$ Hierzu nur ein kleiner Hinweis: Auf eine abstruse Konzeption, nach der Substanzen nackte, all ihrer Eigenschaften entblößbare, Substrate sind, ist Descartes jedenfalls nicht festgelegt. Dies geht z. B. aus seinen Erläuterungen in den Principia (AT VIII-1 28 ff.) hervor: Zwischen einer Substanz und ihrer Haupteigenschaft bestehe nur ein Vernunftunterschied, kein realer, heißt es dort.

Daraus wird erklärlich, warum der Denker, wenn er sich nun der Frage zuwendet, was für eine Art von Substanz er ist, Ausschau danach hält, welche Eigenschaften er sich sogar im Großen Zweifel zubilligen kann und (auch wenn er sich nicht so ausdrückt:) welches seine Haupteigenschaft ( $\approx$ seine Substanz) ist. Die Eigenschaft, ein rationales Lebewesen zu sein, läßt er als zu unklar beiseite. Der Besitz der Eigenschaft, eine Körper-Seele-Einheit zu sein, ist bezweifelbar und mithin nicht das Gesuchte. Auch einige der Eigenschaften, die im Rahmen der aristotelischen Tradition einer Seele zuzuschreiben sind (z. B. Ernährung und Sinnesempfindung), involvieren die Existenz eines Körpers und sind damit keine, deren Besitz dem Denker an diesem Punkt gewiß sein könnte. Allein das Denken könne von ihm nicht losgelöst (d. h. als eine seiner Eigenschaften in Zweifel gezogen) werden, resümiert er. Daran schließt sich die sog. Sum-res-cogitans-Argumentation an:

Ich bin, ich existiere; das ist gewiß. Wie lange aber? Offenbar solange ich denke; denn vielleicht könnte es auch geschehen, daß ich, wenn

$13 \mathrm{Da} ß$ der Substanzbegriff schon lange ausgedient habe und auf die Müllhalde der philosophischen Fehlkonstruktionen gehöre, ist oft zu hören. Zu einer überzeugenden Widerlegung dieses Vorurteils vgl. Schnieder (2004). 
ich mit jedem Denken aufhörte, sofort ganz und gar aufhörte zu sein. Ich gebe jetzt nichts zu als das, was erwiesenermaßen wahr ist; ich bin also genau nur eine Sache, die denkt [...] Ich bin aber eine wahre und wahrhaft existierende Sache. Was für eine Sache? Ich sagte es: eine denkende. (AT VII 27)

Diese vieldeutige Passage läßt sehr unterschiedliche Auslegungen zu, insbesondere hinsichtlich dessen, wie weit mit ihr der Beweis dafür vorbereitet sein soll, daß Denken die wesentliche, und darüber hinaus: die einzig wesentliche, Eigenschaft des Meditierenden ist. ${ }^{14}$ Es ist hilfreich, hier eine Unterscheidung von Schütt $(1990,192)$ heranzuziehen: die zwischen realer und epistemischer Essenz. Für unsere Zwecke mag folgende Erläuterung ausreichen: Eine Eigenschaft $E$ gehört zur realen Essenz des Denkers, wenn er nicht existieren kann, ohne $E$ zu haben; und $E$ gehört zu seiner epistemischen Essenz, wenn er nicht daran zweifeln kann, daß er $E$ hat. Als Kern der zitierten Passage läßt sich damit folgendes herausschälen: Zum epistemischen Wesen des Denkers gehört, seit dem Existo-Argument, das Denken und (bisher) nur das Denken; von jeder anderen Eigenschaft kann er (noch) bezweifeln, daß er sie hat. Und dieses Ergebnis verdankt sich dem Existo-Argument. Denn das, dessen Existenz der Denker darin als unbezweifelbar eingesehen hat, ist etwas, das in der gewißheitsstiftenden Einsicht ausschließlich dadurch bestimmt ist, daß es denkt.

Descartes hebt ausdrücklich hervor, daß an dieser Stelle die reale Essenz des Denkers noch nicht thematisiert wird. Er schließt nicht aus, daß körperliche Eigenschaften , in der Wahrheit der Sache“ zu ihm, zu seiner realen Essenz, gehören (AT VII 27). Entscheidend ist hier einzig, daß sie jedenfalls nicht zu seiner epistemischen Essenz gehören. Anders gesagt, hier wird ein vorläufiges, rein negatives Ergebnis erreicht: Dazu, wie er sich selbst an diesem Punkt seiner Überlegungen klar und deutlich begreift, gehört nicht, daß er sich als eine Substanz begreift, die körperliche Eigenschaften hat. Erst in der Sechsten Meditation (AT VII 78) wird die erheblich stärkere positive These vertreten, der Denker begreife sich klar und deutlich als eine Sache, die keine körperlichen Eigenschaften hat.

Eine denkende Substanz bezeichnet Descartes als Geist (und setzt dies synonym mit: Verstandesseele [animus], Intellekt oder Vernunft). Das Verständnis, das der Denker über sich selbst erreicht hat, läßt sich nun so charakterisieren: Was auch immer er selbst sonst noch sein mag, jedenfalls ist er etwas, dessen Existenz ihm durch das Existo gewiß ist. Soweit er

14 Eine umfangreiche und sorgfältige Studie zu den Mehrdeutigkeiten und den zahlreichen Rekonstruktionsversuchen in der Sekundärliteratur hat S. Dierig (2003) vorgelegt. 
sich selbst genau als das nimmt, von dessen Existenz er Gewißheit hat, ist er ausschließlich eine denkende Sache, ein Geist, ein Intellekt. Er verfügt jetzt über einen neuen Sinn von „ich“. Dies ist nicht der naiv körpergetränkte und verschwommene Sinn, den er mit diesem Wort verband, bevor er sich in den Großen Zweifel begab. Und es ist auch nicht der unnatürlich ausgeklügelte Sinn, den er mit dem Wort „ich“ verbinden mußte, um dann im Großen Zweifel Gewißheit über seine eigene Existenz zu erlangen. Vielmehr ist es ein einfacher und präziser Sinn von „ich“, den er sich jetzt erst erschlossen hat, und zwar: der Sinn von „der, dessen Existenz (durch das Existo-Argument) gewiß ist" [ille ego quem novi, AT VII 27]. ${ }^{15}$ Und in diesem Sinne ist er, soweit er bisher weiß, nichts anderes als das, was er von nun an mit „mein Geist" bezeichnet: diejenige denkende Substanz, deren Existenz ihm durch das Existo unbezweifelbar ist.

Der Denker ist jedenfalls ein Geist; ob er auch noch etwas Darüberhinausgehendes ist, kann er weder mit Gewißheit bejahen, noch mit Gewißheit verneinen. Stattdessen versucht er genauer zu verstehen, was ein Geist ist.

\section{Unvorstellbarkeit und Einheit des Geistes}

Eine Frage, die Descartes damit zugleich aufwirft, ist: Mit Hilfe welches Vermögens läßt sich denn überhaupt ein solches Verständnis erreichen? Seine Untersuchung nimmt hier wieder eine Leitfrage der Meditationen auf: die nach der kognitiven Leistungskraft der einzelnen menschlichen Erkenntnisvermögen. ${ }^{16}$ Keine Auskunft, die sich auf Sinneswahrnehmung oder Gedächtnis berufen müßte, darf der Denker heranziehen, um besser zu verstehen, wer er ist. Aber auch das Vermögen der bildlichen Vorstellung könne zu diesem Verständnis nichts beitragen; denn Vorstellungsbilder seien Bilder von körperlichen Sachen und folglich sei alles, was sie darstellen, dem Zweifel ausgesetzt und könne mithin nicht der Geist sein,

15 Setzt dieser Sinn von „ich“ nicht einen anderen Sinn von „ich“ voraus? Denn deutlicher müßte es doch heißen: „der, dessen Existenz mir (durch das Existo-Argument) gewiß ist“. Doch darin liegt keine Schwierigkeit. Seit dem Existo-Argument verfügt der Denker über einen geeigneten anderen Sinn von „ich“, nämlich der Sinn von „der Denker dieses Gedankens“.

16 Perler (in diesem Band, 26) spricht mit Hinblick auf die Erste Meditation von einem Vermögensskeptizismus, den Descartes strategisch einsetze. In der Zweiten Meditation wird solch ein Skeptizismus noch nicht endgültig überwunden. Aber schon hier klingt die Sonderstellung des Intellekts unter den menschlichen Erkenntnisvermögen an, deren ganzes Ausmaß erst in der Sechsten Meditation deutlich wird. Vgl. dazu auch Hatfield (in diesem Band, 127 ff.). 
dessen Existenz jetzt unbezweifelbar ist. Kurz, der Geist ist unvorstellbar. Nach der Sinneswahrnehmung und der Erinnerung erweist sich damit nun auch das Vorstellungsvermögen als grundsätzlich unbrauchbar, um zu verstehen, was der eigene Geist ist. ${ }^{17}$ Allein der reine Intellekt bleibt als ein Vermögen, mit dem sich dies verstehen läßt. Daß Sinneswahrnehmung und Vorstellungsvermögen nicht nur zur Erkenntnis des eigenen Geistes unbrauchbar sind, sondern sogar zur Erkenntnis des Wesens der körperlichen Dinge nicht taugen, ist ein Ergebnis, das durch die nachfolgende Betrachtung untermauert werden soll, die ich hier beiseitelasse (AT VII 30-33).

Bei seiner Untersuchung dazu, welches seine wesentlichen Eigenschaften sind, soweit er ein Geist ist, verdeutlicht Descartes zunächst einmal, in welch weitem Sinn er das Wort „denken“ gebraucht: Jedes Haben von Gedanken ist Denken. Ob man einen Gedanken in der Weise hat, daß man an seiner Wahrheit zweifelt, oder in der, daß man seinen Inhalt als wahr einsieht, oder in der, daß man seinen Inhalt verneint - all das macht keinen Unterschied im Hinblick darauf, daß man dabei denkt. Zweifeln, Begreifen, Zustimmen, Verneinen, - ja, Wollen, Vorstellen und sinnliches Empfinden sind Arten des Denkens. Zwar gibt es mannigfache Arten des Gedankenhabens, aber es ist immer ein und dieselbe Substanz, die die Gedanken des Denkers hat (AT VII 29, 86). Der Geist des Denkers besteht nicht aus separaten Teilgeistern, die unterschiedliche Tätigkeiten ausführen, sondern er ist eine einzige Geist-Substanz, die bei der Ausführung ihrer verschiedenen Tätigkeiten immer eines tut: Sie denkt. Seien seine Gedanken bzw. die Art, in der er sie hat, noch so verschieden, es ist ein und derselbe Geist, der sie hat.

Diese These von der synchronen Einheit des Geistes macht Descartes zwar explizit, aber er gibt keine Begründung für sie. Sie sei so offenkundig, daß er sie nicht durch irgend etwas noch Evidenteres erklären könne. - Es sei nebenbei angemerkt, daß Descartes eine These von der diachronen Identität des Geistes in der Zweiten Meditation nicht einmal formuliert, ${ }^{18}$ geschweige denn verteidigt, obwohl er seinen Denker offenbar voraussetzen läßt, daß es ein und derselbe Geist ist, der über die Zeit hinweg all die Gedanken hat, an denen er uns teilhaben läßt.

17 Eine geistvolle Vorstellung davon, was herauskommen kann, wenn ein Geist, mit durchaus cartesianischen Neigungen in der Metaphysik, versucht, sich sich selbst vorzustellen, gibt Beckett (1938, Kap. 6).

18 Er nennt sie allerdings in der Synopsis zur Zweiten Meditation (AT VII 14) und erklärt, er habe sie in den Meditationen deswegen nicht behandelt, weil ihre Begründung von einer Erläuterung der gesamten Physik abhänge. 


\section{Das Geistige am Sinnlichen}

Um Sinnesempfindungen zu haben, bedarf es entsprechender Sinnesorgane, also eines Körpers. Das Charakteristikum menschlicher Sinnesempfindung, im Gegensatz zu der von Tieren, ist für Descartes jedoch, daß zu ihr das Denken gehört (AT V 277). Doch wie kann er auch Empfindungen - zumal solche, die dem Körper geschuldet sind - zum Denken rechnen?

Descartes gibt folgende Begründung für seine Auffassung. Zum Sehen, Hören und Fühlen gehöre es, daß es dem Subjekt so scheint, als sehe, höre und fühle es etwas. Dieses So-Scheinen ist für Descartes ein geistiger Aspekt sinnlichen Empfindens - ja, er nennt diesen Aspekt das eigentliche sinnliche Empfinden, das genau so verstanden nichts anderes sei als ein Denken. ${ }^{19} \mathrm{Da} ß$ er ein Licht sieht, muß dem Denker in seinem Großen Zweifel als falsch gelten, selbst wenn es ihm so scheint, als sehe er eines. Aber daß es ihm so scheint, als sehe er etwas, könne dann nicht falsch sein (AT VII 29).

Diese Begründung ist oft so verstanden worden, als vertrete Descartes eine abwegig über-intellektualistische Konzeption: Eine Sinnesempfindung haben, heiße, einen Gedanken haben, der sich sprachlich wiedergeben läßt als „Es scheint mir, daß ich ein Licht sehe“. ${ }^{20}$ Und dieser Gedanke könne eben wahr sein, auch wenn die von „Ich sehe ein Licht“ oder „Da ist ein Licht" ausgedrückten Gedanken falsch sind. Danach wäre das, was Descartes die eigentliche Sinnesempfindung nennt, nichts anderes als ein sei's auch besonders vorsichtiges Urteil - mithin ein geistiger Akt, der auch dann vollzogen werden und dessen Inhalt auch dann wahr sein kann, wenn es keine körperlichen Dinge gibt. Doch es gibt Grund anzunehmen, daß dies nicht Descartes' Auffassung ist. Sinnesempfindungen sind keine Urteile. ${ }^{21}$ Urteile sind, wie später in den Meditationen deutlich wird, Tätigkeiten, an denen Intellekt und Wille beteiligt sind. Hingegen sind die ,eigentlichen' Sinnesempfindungen, streng genommen, überhaupt nichts, das dem Intellekt (und dem Willen) zuzurechnen ist. Das geht aus den ausführlicheren

19 Mit dem Mir-so-Scheinen geht es, wie wir sehen werden, Descartes um eine besondere Form des Denkens, die sich vom Denken des reinen Intellekts unterscheidet. - Zu einer anderen Deutung, wonach Descartes Denken schlechthin mit dem Mir-so-Scheinen gleichsetzt, siehe A. Koch (2004, 45 ff.).

20 So z. B. N. Malcolm (1977, 45 ff.).

21 Obwohl Descartes (leider) von den Urteilen der äußeren und inneren Sinne spricht (AT VII 76 f.). Es wird im Zusammenhang jedoch klar, daß er voreilige Urteile meint, die allzu unkritisch auf Grund von Sinnesempfindungen getroffen werden. 
Erörterungen zu diesem Thema hervor, die sich in der Sechsten Meditation und in den Sechsten Erwiderungen finden (AT VII 86 ff. und 436 f.). Sinneswahrnehmung begreift Descartes als einen komplexen Prozeß, in dem sich drei Stufen unterscheiden lassen: erstens die rein körperliche Stufe der bis ins Gehirn reichenden Nervenreizung, zweitens die geistige (aber noch nicht intellektive) Stufe der undeutlichen Perzeption eines nicht-reinen Gedankens (AT III 493), der unmittelbar von der Bewegung im Gehirn bewirkt wird, und schließlich die intellektive Stufe der Bildung eines reinen Gedankens, der auf Grund des nicht-reinen Gedankens gebildet wird und dessen Inhalt der eines Wahrnehmungsurteils sein kann. Was Descartes in der Zweiten Meditation als, die eigentliche Empfindung' bezeichnet, ist das, was sich auf der zweiten Stufe abspielt. Es ist das Ereignis, in dem eine Bewegung im Gehirn dem Geist ein Zeichen gibt [menti signum dat, AT VII 88], etwas zu empfinden. Das im Geist empfangene Zeichen ist eine Idee, mithin ein Gedanke (AT VII 38). Aber es ist kein reiner Gedanke des Intellekts. Vielmehr handelt es sich um einen Gedanken, dessen Inhalt nicht begrifflich, sondern, wie man vielleicht sagen kann, sinnlich ist. Aus dem sinnlichen Inhalt dieser nicht-begrifflichen und mithin nicht wahrheitswertfähigen Empfindung errechnet [ratiocinari, AT VII 437] der Intellekt Ergebnisse mit einem Inhalt, der wahr oder nicht-wahr ist und gewöhnlich von der Beschaffenheit der körperlichen Ursachen der Empfindung handelt. Es ist für Descartes offenbar der Witz ,eigentlicher Empfindungen, zu propositionalen Gedanken zu führen. Aber erst aus den Aktivitäten des Intellekts ergeben sich wahrheitswertfähige Gedanken, deren Inhalt der eines Urteils werden kann.

Seit frühester Kindheit sind wir es gewohnt, solche Urteile sehr rasch und unkritisch zu bilden (AT VII 438); das ist für unser Überleben wichtig, führt aber nicht selten zu Irrtümern: Wir urteilen „Da ist ein Licht“ oder „Ich sehe ein Licht“, auch wenn durch die Empfindung, die wir haben, nur die Wahrheit von „Es scheint mir, daß da ein Licht ist“ bzw. „Es scheint mir, daß ich ein Licht sehe“ verbürgt ist. - Nach diesem Verständnis von Descartes' Theorie der Sinneswahrnehmung ist die ,eigentliche' Sinnesempfindung so etwas wie ein (im Hinblick auf Wahrheit und Falschheit) noch schlummernder Gedanke, aber doch schon ein Gedanke. Jedoch erst durch einen Akt des Intellekts, auf den die Empfindung, in ihrem Schlummer' nur abzielt, entsteht etwas rein Geistiges. ${ }^{22}$ Eine ,eigentliche Empfin-

22 Tiere haben für Descartes keine Empfindungen, insofern sie keine, eigentlichen' Empfindungen haben; und die haben sie seines Erachtens nicht, weil nichts dafür spreche, daß sie einen Intellekt haben. - Tierquäler können sich deswegen nicht auf Descartes berufen. Vieler- 
dung ist für Descartes zwar ein Gedanke, aber nicht ein propositionaler, der von „Es scheint mir ..."-Sätzen ausgedrückt wird, sondern ein nichtpropositionaler, der solche Sätze wahr werden läßt. ${ }^{23}$

Daß Descartes Sinnesempfindungen als Gedanken konzipiert, ist also keineswegs ein Hinweis auf eine übermäßig intellektualisierende Theorie kreatürlicher Sinnlichkeit, sondern macht besonders deutlich, wie einschneidend er den üblichen Gebrauch von „Gedanken haben“ erweitert, damit Denken ein plausibler Kandidat für die gesuchte Haupteigenschaft des Geistes sein kann. Es gibt für ihn Gedanken, deren Inhalt nichts Begriffliches ist, erst recht keine Proposition. ${ }^{24}$ Nicht alles, was in Descartes' Sinn von „denken“ gedacht wird, läßt sich mit einem Daß-Satz wiedergeben. Das Geistige am Sinnlichen, das sind undeutliche Gedanken ohne begrifflichen Gehalt; erst der Intellekt gelangt durch Schlußfolgerungen zu Gedanken im üblichen Sinne.

Doch welchen Grund gibt es für Descartes, den Begriff des Denkens in dieser Weise auszuweiten? Oder hat er gar keinen sachlichen Grund, sondern nur das gerade erwähnte Motiv, Denken als Haupteigenschaft des Denkers erscheinen zu lassen? Dann wäre diese Erweiterung nichts als eine Art Etikettenschwindel, wie ihm dies ja auch gelegentlich vorgeworfen wird: eine Stipulation, mit der Einheitlichkeit terminologisch vorgespiegelt wird, obwohl die Phänomene disparat und heterogen sind. Oder hat eine Schmerzempfindung, zum Beispiel, etwas an sich, dank dem es gerechtfertigt wäre, sie als einen Gedanken zu bezeichnen? - Eine Antwort, die einzige, die Descartes meines Wissens anbietet, findet sich in seiner Definition von „Gedanke“ (AT VII 160), wonach er mit diesem Wort alles bezeichnet, das so in uns ist, daß wir uns seiner unmittelbar bewußt sind. Im Lichte dieser Worterläuterung ist es in der Tat gerechtfertigt, Empfindungen zu den Gedanken zu rechnen: Vieler Dinge (z. B. der Verletzung unseres Fußes) sind wir uns bewußt, aber nicht unmittelbar bewußt. Der Schmerzempfindung hingegen, dank der wir uns der Verletzung unseres

lei Leiden bedarf keiner ,eigentlichen' Empfindung; Tiere haben seines Erachtens eine körperliche Seele und ein organisches Sinnesempfinden (AT VII 426). Siehe dazu Kemmerling (1996, 211-217).

$23 \mathrm{Zu}$ dem exegetisch sehr diffizilen Thema des Inhalts von Sinnesempfindungen und der Nicht-Propositionalität mancher Gedanken siehe Alanen (2003, Kap. 2-Kap. 5), Haag (2008), Kemmerling (1996, 52-76), Perler (1996, 48-64) und Wilson (1999, Kap. 2-Kap. 5).

24 Descartes uneingeschränkt als einen Propositionstheoretiker des Inhalts von Gewißheit zu bezeichnen, wie P. Markie (1986, 73 ff.) das tut, ist demnach falsch. Nach Descartes sind wir uns auch unserer ,eigentlichen' Sinnesempfindungen bewußt; aber diese sinnliche Gewißheit hat keinen propositionalen Inhalt. 
Fußes bewußt sein können, sind wir uns unmittelbar bewußt: Es verdankt sich nichts anderem als ihr selbst, daß wir uns ihrer bewußt sind.

Ist mithin unmittelbares Bewußtsein für Descartes das Wesensmerkmal des Denkens und damit des Geistes? Gary Hatfield $(2003,124)$ hat zurecht darauf aufmerksam gemacht, daß die gerade erwähnte Definition keine Beschreibung des Wesens einer denkenden Sache ist, sondern eine Charakterisierung der Extension des Wortes „Gedanke“. Und es gibt eine Reihe von Gründen gegen die Annahme, unmittelbares Bewußtsein sei für Descartes das Wesen der geistigen Substanz. Zunächst einmal wäre zu erwarten, daß er dies in der Zweiten Meditation gesagt hätte; aber in ihr ist von Bewußtsein gar nicht die Rede, insbesondere auch dann nicht, wenn der Denker durch Synonyme verdeutlicht, was er unter einer denkenden Substanz versteht: Geist, Verstandesseele, Intellekt, Vernunft. - Wichtiger ist jedoch, daß Descartes einzig den Intellekt als dasjenige geistige Vermögen erachtet, das einem Geist nicht abgehen kann. In der Sechsten Meditation läßt er seinen Denker dies deutlich aussprechen: Das Vorstellungsvermögen gehöre nicht zum Wesen seines Geistes [mentis meae essentia, AT VII 73]. Er könne sich auch dann klar und deutlich als ein Ganzes begreifen, wenn ihm das Vermögen der bildlichen Vorstellung und das der Sinnesempfindung fehlten; aber diese beiden Vermögen könne er nicht begreifen, außer als ihm innewohnend - ihm, das heiße: einer begreifenden Substanz [substantia intelligens, AT VII 78].

Eine spezifischere Auskunft über das Wesen des Geistes als die, er sei eine denkende Substanz, wird erst in der Secbsten Meditation explizit formuliert: Der Geist ist seinem Wesen nach eine begreifende, d. h. begrifflich denkende, Substanz. ${ }^{25}$ In der Zweiten Meditation wird dieses Ergebnis nur andeutungshaft vorbereitet, zum einen durch die erwähnte Liste von Synonymen, zum andern durch die Schlußüberlegung, der wir uns nun zuwenden wollen.

\section{„Über die Natur des menschlichen Geistes: daß er besser bekannt ist als der Körper“"}

Dem Thema, das die Überschrift der Zweiten Meditation verheißt, wendet sich Descartes erst ganz an ihrem Ende, und dort in verblüffender Kürze, zu. In einer Zwischenbetrachtung ließ er den Denker zu dem Ergebnis gelangen, daß er das Wesen (im Gegensatz zu den akzidentellen Eigen- 
schaften) eines konkreten körperlichen Dings, eines Stücks Wachs, weder mit Hilfe der Sinneswahrnehmung noch mit Hilfe des bildlichen Vorstellungsvermögens, sondern allein mit dem Intellekt erkennen könne. „Was bin ich“, fragt er sich, „der ich dieses Wachs so deutlich zu perzipieren scheine?" (AT VII 33).

Durch zweierlei möchte er sich besser begreiflich machen, was er ist: durch die Einsicht, daß er sich selbst, erstens, „viel wahrer und gewisser“ und, zweitens, „viel deutlicher und evidenter“ erkennt als jeden körperlichen Gegenstand, den es geben mag, z. B. ein Stück Wachs. Die Überlegung zur Stützung des ersten Punkts ist folgende: Aus jedem Grund, den er dafür haben könnte zu schließen, daß ein bestimmter körperlicher Gegenstand existiert, folgt noch viel evidenter, daß er selbst existiert. Denn aus jedem Gedanken von der Art, daß er ein Stück Wachs sieht oder zu sehen denkt, berührt oder zu berühren denkt, usw. folgt seine eigene Existenz zwingend, nicht aber die des Wachses. Wie gut auch immer ein Grund sein mag, den der Denker dafür hat, daß ein bestimmter körperlicher Gegenstand existiert, es ist unweigerlich ein noch viel besserer Grund, sich der eigenen Existenz gewiß zu sein. - Dieses Argument ist enttäuschend, denn die Einsicht, daß der Denker in dieser Weise auf die eigene Existenz schließen kann, ist ja nicht neu. Als Beitrag zur Antwort auf die Frage, was das Wesen, die Natur, seiner selbst als einer denkenden Substanz ist, wirkt diese Überlegung schlicht irrelevant. Nicht nur philologisch, sondern vielleicht auch philosophisch bemerkenswert ist allerdings, daß von Natur oder Wesen in diesem Textstück gar nicht mehr die Rede ist, obwohl es um die Frage geht „Was bin ich?“.

In dieser Hinsicht verspricht die zweite Überlegung mehr, insofern in ihr immerhin ausdrücklich von der Natur des Geistes die Rede ist. Die These, die darin begründet werden soll, ist offenbar folgende: Die Anzahl der Eigenschaften seines eigenen Geistes, die der Denker kennt, ist auf jeden Fall größer als die der Eigenschaften aller Körper, die er kennen könnte. Im Lichte dessen, was Descartes in den Fünften Erwiderungen ausführt, ist das Argument für diese These folgendermaßen zu verstehen: (1) Zu jeder beliebigen körperlichen Eigenschaft $K$, die dem Geist erkennbar ist, gibt es die ihm ebenfalls erkennbare geistige Eigenschaft $G_{K}$, dazu befähigt zu sein, $K$ zu erkennen. (2) Darüber hinaus sind dem Geist noch beliebig viele andere Eigenschaften seiner selbst $\left(G_{x}, \ldots, G_{y}\right)$ bekannt, die nichts mit der Wahrnehmung körperlicher Gegenstände zu tun haben. Also (3) sind dem Geist weit mehr Eigenschaften seiner selbst ( $G$-Eigenschaften) erkennbar als Eigenschaften beliebiger anderer Dinge ( $K$-Eigenschaften). Folglich (4) ist die Natur des Geistes die ihm von allen Substanzen am besten bekannte [natura ... notissima, AT VII 360]. 
Nun ja, das sieht scharfsinnig aus, aber Eigenschaftszählerei ist eine heikle Sache. Wenn die erkannten $G$-Eigenschaften und die erkannten $K$-Eigenschaften abzählbar unendlich viele sind (und dies anzunehmen ist plausibel) dann hat es keinen guten Sinn zu sagen, es gebe von den einen "mehr" als von den andern. Von diesem Argument ließe sich wohl nur einer beeindrucken, der auch bereit ist zu glauben, es gäbe „mehr“ natürliche Zahlen als Primzahlen.

Gassendi hat in seinen Einwänden gegen die Zweite Meditation herausgearbeitet, inwiefern diese Überlegung auch in anderer Hinsicht enttäuschend ist (AT VII 275 ff.). Sein Einwand läßt sich so paraphrasieren: Die Natur einer Substanz ist nur durch solche tieferen Struktureigenschaften charakterisiert, die eine Erklärung dafür erlauben, weshalb diese Substanz die für sie typischen Oberflächeneigenschaften hat. Die Natur des Weines z. B. besteht nicht darin, daß er rot oder weiß, flüssig, berauschend usw. ist, sondern in seinen tieferen chemischen Eigenschaften, aus deren Zusammenspiel solcherlei Oberflächeneigenschaften resultieren. Was Descartes in seiner zweiten Überlegung präsentiert, ist jedoch schlicht eine wahllose Vielfalt von Oberflächeneigenschaften der denkenden Substanz. Soweit Gassendis Einwand. - Noch enttäuschender als Descartes' ursprüngliche Überlegung selbst wirkt seine aggressiv polemische Erwiderung auf diesen Einwand (AT VII 359 f.), in der er die Unterscheidung zwischen Oberflächen- und Struktureigenschaften schlicht ignoriert und seine krude quantitative Konzeption der Wesenskenntnis mit bissigem Nachdruck wiederholt: ,Je mehr Eigenschaften einer Substanz wir erkennen, desto vollkommener begreifen wir ihre Natur" (AT VII 360).

Gibt es ein Verständnis dieser prima facie unbefriedigenden Auskunft über die Natur des Geistes, in dessen Licht sie weniger enttäuschend ist? Ich denke, das gibt es. Zunächst sollte man einen Hinweis im Text ganz ernstnehmen: den genauen Wortlaut der Überschrift zur lateinischen Fassung der Zweiten Meditation. Sie lautet eben nicht (wie der Duc de Luynes sie schon in der französischen Übersetzung wiedergibt): „Über die Natur des menschlichen Geistes; \& daß er besser bekannt ist als der Körper“. Im lateinischen Text ist da kein Semikolon und kein „\&“, stattdessen ein aufschlußreicher Doppelpunkt, der die Natur des Geistes und sein besseres Bekanntsein in einen engen Zusammenhang bringt. Man beachte auch, wie völlig selbstverständlich Descartes seinen Denker an der betreffenden Stelle (AT VII 25/26) von der Frage, was er sei, zu der Frage übergehen läßt, ob er sich selbst nicht in mannigfachen Hinsichten besser erkenne als jenes Stück Wachs, das stellvertretend für jeden beliebigen Körper betrachtet wurde. Angesichts dessen könnte, was in dieser Medita- 
tion über die Natur des menschlichen Geistes gezeigt werden soll, dieses sein:

„Die Natur des menschlichen Geistes zeigt sich am deutlichsten daran, daß er besser - und zwar in jeder epistemisch relevanten Hinsicht besser - bekannt ist als der Körper. Wir erkennen den Geist mit Hilfe des reinen Intellekts früher ${ }^{26}$, wahrer, gewisser, deutlicher, evidenter, umfassender und leichter als jeden Körper, und gerade dies gibt uns Aufschluß darüber, was für eine ganz und gar andere Art von Substanz er ist. Schon die für Gassendi völlig selbstverständliche Vorstellung, es müsse auch im Hinblick auf die Eigenschaften des Geistes eine Hierarchie geben: von den oberflächlichen, leicht zu bemerkenden, aber erklärungsbedürftigen, bis hinab zu den tiefsten, am schwersten zu entdeckenden, aber dafür erklärungsstärksten, schon diese Vorstellung ist ein profundes Mißverständnis. Der Geist ist etwas ganz und gar anderes. Er hat kein Zentrum und keine Peripherie, weder Tiefe noch Oberfläche. Er hat kein Inneres, nichts an ihm ist sich selbst verborgen. Wer verstanden hat, wie grundsätzlich die Erkenntnis des Geistes sich von der eines körperlichen Dings unterscheidet, der hat auch die Natur des Geistes begriffen. Und er sieht dann auch, daß eine wesenserforschende Wissenschaft des Geistes nicht nur überflüssig, sondern unmöglich ist." 27

Es scheint nicht völlig abwegig anzunehmen, daß etwas dieser Art Descartes' Auffassung über die Natur des Geistes sein könnte.

\section{Literatur}

Alanen, Lilli 2003, Descartes's Concept of Mind, Cambridge, Mass.

Beckett, Samuel 1938, Murphy, London

Carriero, John 1986, The Second Meditation and the Essence of the Mind, in: Essays on

Descartes' Meditations, hrsg. v. A. O. Rorty, Berkeley, 199-221

Dicker, Georges 1993, Descartes. An analytical and historical introduction, New York

26 Die Existenz des eigenen Geistes läßt sich, wie wir gesehen haben, sogar erkennen, bevor klar ist, was sein Wesen ist. Dies unterscheidet ihn wesentlich von allen andern Substanzen. Gottes Existenz und auch die materieller Körper wird in den Meditationes erst erkannt, nachdem ihr Wesen bestimmt wurde.

$27 \mathrm{Zu}$ Argumenten für die These, daß im Rahmen der Cartesischen Philosophie für eine Wissenschaft des Geistes oder der Körper/Geist-Beziehung kein Platz ist, vgl. Wilson (1978, 98 f. und 164 f.) und Alanen (2003, 53 und 72 ff.). 
Dierig, Simon 2003, Sum res cogitans und der Substanzendualismus von Körper und Geist, München

Dretske, Fred 2003, Knowing what You Think vs. Knowing that You Think It, in: The Externalist Challenge: New Studies in Cognition and Intentionality, hrsg. v. R. Schantz, Berlin, 389-399

Frankfurt, Harry G. 1970, Demons, Dreamers, and Madmen: the Defense of Reason in Descartes's Meditations, Princeton

Gaukroger, Stephen 1995, Descartes. An Intellectual Biography, Oxford

Haag, Johannes 2008, Sinnliche Ideen - Descartes über sinnliche und begriffliche Aspekte der Wahrnehmung, in: Sehen und Begreifen - Wahrnehmungstheorien in der frühen Neuzeit, hrsg. v. D. Perler/M. Wild, Berlin, 95-121

Hatfield, Gary 2003, Descartes and the Meditations, London

Hatfield, Gary 2009: The Sixth Meditation: Mind-Body Relation, External Objects, and Sense Perception, in diesem Band, Kap. 7

Hüttemann, Andreas 2009: Die Grundlegung der Cartesischen Physik in den Meditationen, in diesem Band, Kap. 9

Kemmerling, Andreas 1996, Ideen des Ichs, Frankfurt/M., Seitenangaben beziehen sich auf die 2. Auflage 2005, Frankfurt/M.

Kenny, Anthony 1968, Descartes. A Study of His Philosophy, New York

Koch, Anton 2004, Subjekt und Natur-Zur Rolle des „Ich denke“ bei Descartes und Kant, Paderborn

Lichtenberg, Georg Christoph 1969, Schriften und Briefe, Band 2, hrsg. v. W. Promies, München

Malcolm, Norman 1977, Thought and Knowledge, Ithaca

Markie, Peter 1986, Descartes's Gambit, Ithaca

- 1992, The Cogito and its Importance, in: The Cambridge Companion to Descartes, hrsg. v. J. Cottingham, Cambridge, 140-173

Nietzsche, Friedrich 1968, Werke, Kritische Gesamtausgabe, Bd. VI-2, hrsg. v. G. Colli und M. Montinari, Berlin

- 1970, Werke, Kritische Gesamtausgabe, Bd. VII-3, hrsg. v. G. Colli und M. Montinari, Berlin

Perler, Dominik 1996, Repräsentation bei Descartes, Frankfurt/M.

- 2009, Strategischer Zweifel - Die Funktion skeptischer Argumente in der Ersten Meditation, in diesem Band, Kap. 2

Russell, Bertrand 1945, A History of Western Philosophy, New York

Schnieder, Benjamin 2004, Substanzen und (ihre) Eigenschaften, Berlin

Schütt, Hans-Peter 1990, Substanzen, Subjekte und Personen. Eine Studie zum Cartesischen Dualismus, Heidelberg

- 2009, Die Stellung der Meditationen im Gesamtwerk Descartes', in diesem Band, Kap. 8

Specht, Rainer 1996, Pragmatische Aspekte der cartesischen Metaphysik, in: Descartes nachgedacht, hrsg. v. A. Kemmerling/H.-P. Schütt, Frankfurt/M., 6-23

Wilson, Margaret D. 1978, Descartes, London

- 1999, Ideas and Mechanism - Essays on Early Modern Philosophy, Princeton 